\section{Investigating Persistent Bilateral Ankle Swelling}

\section{Abstract}

This paper presents a case-report of a young female reporting sudden onset ankle swelling and pain, subcutaneous inflammation and progressive bilateral pes planus, in the context of no associated co-morbidities or risk factors for autoimmune and infective conditions. We discussed the appropriate imaging techniques which facilitated the diagnosis, which included Xrays, ultrasound and MRI scans used to rule out soft tissue injuries, stress fractures, inflammatory arthritis, and other causes of peripheral nerve entrapment and tendon pathology. The patient was diagnosed with polyarteritis nodosa based on deep skin biopsy. This is the first case ever reported showing significant peripheral nerve enlargement in the context of small vessel vasculitis.

The imaging step-up approach is discussed in relation to the sensitivity and specificity of different imaging techniques for assessing joint, bone, tendon, ligament and nerve-related pathology. The rarity of this diagnosis in the context of a very common presentation emphasised the need for specialist referral and has significant educational value.

\section{Introduction}

\section{Learning points}

- Any patient with persistent joint swelling should be referred to the rheumatologist/general physician/orthopaedic surgeon for further investigations.

- Full history and multi-system clinical examination along with blood tests help in deciding the most appropriate imaging technique required for diagnosis.

- Ultrasonography and magnetic resonance imaging (MRI) are the techniques of choice for evaluating ankle inflammatory conditions. Computer tomography (CT) could add value in assessing for struc-
Coziana Ciurtin'1, Gayani Pitiyage ${ }^{2}$ Petrie de Villiers ${ }^{3}$, Paul Glynne 4

1 Department of Rheumatology, University College London NHS Trust, 3rd Floor Central, 250 Euston Road, London.

2 Department of Pathology, Rockefeller Building, University Street, London.

3 Department of Radiology, University College London NHS Trust, London, UK, 235 Euston Road, London.

4 Department of Medicine, University College London NHS Trust, London, UK, 235 Euston Road, London.

\section{Contact information:}

\section{Coziana Ciurtin.}

Department of Rheumatology.

Address: University College London NHS Trust, 3rd Floor Central, 250 Euston Road, London, NW1 2PG, UK.

Tel: 44(0)2034479035.

\section{c.ciurtin@ucl.ac.uk}

\section{Keywords}

Tibialis Posterior Nerve Enlargement; Polyarteritis Nodosa; Ankle Swelling of Rare Causes. 
tural abnormalities but has lower sensitivity for the detection of soft tissue inflammation.

- In selected cases, histological examination of the abnormal structures identified by imaging is required for diagnosis.

\section{Patient}

A 38 years old woman presented with acute onset pain followed by bilateral ankle swelling. Her symptoms got progressively worse during a period of a few weeks but she didn't report any initial injury. She also noticed progressive bilateral ankle deformity with collapsed arches, bluish discolouration and swelling over both the lateral and medial malleoli and associated tingling and numbness in her feet. She denies any relevant personal or family medical history.

Clinical examination showed diffuse swelling across the both ankles with associated skin discolouration with bluish appearance. There was also evidence of pes planus upon standing, but this corrected on double tip toes. The "too many toes" sign was positive bilaterally, as the front of her feet pointed outwards, suggesting posterior tibial tendon (PTT) dysfunction. The patient had moderate pain over both malleoli with a strongly positive Tinel's sign/test over the distribution of the tibial nerve just posterior to the medial malleolus. The ankle mobility was reasonably well preserved bilaterally. No systemic cause for the bilateral ankle inflammation was identified at the clinical examination. Blood tests were normal, apart from moderately increased inflammatory markers with negative screening for infective causes and no autoimmune abnormalities.

\section{What is the next investigation?}

Ankle swelling may be the result of a systemic, regional or localised disease. It is one of the most common clinical pictures that presents to general practitioners, rheumatologists and general physicians, as an isolated complaint or in the context of various comorbidities. Investigations should be directed at excluding systemic causes associated with fluid retention and assessing the cause of localised ankle inflammation. Because the differential diagnosis of ankle swelling is very broad, clinicians should be aware of the most appropriate imaging techniques that can be employed for diagnosis. The absence of systemic features in our patient's presentation, directed the investigation towards the exclusion of ankle soft tissue injuries and inflammation of different causes. Bilateral ankle fracture or soft tissue injury is rarely seen, mainly in the context of significant trauma.

\section{Radiography}

Plain radiographs of the ankle in two different projections are recommended to exclude fractures or dislocations and other gross abnormalities [1]. The radiographs can also detect soft tissue swelling but are less useful in assessing for the cause of inflammation. Because of the pain and bone tenderness over the posterior aspect of the medial malleolus, according to the Ottawa ankle rules [2], the radiography of the ankle was recommended to exclude fractures. Our patient's plain radiographs showed no significant abnormalities (Figure 1). When no abnormalities were visible on radiographs, further imaging techniques should be employed, according to the clinical examination and local policies.

\section{Ultrasonography}

This non-radiative, observer-dependent technique also enables the dynamic assessment of the ankle tendons and ligaments. US has high sensitivity and specificity (above 89\%) in detecting ankle ligament injuries [3] and similar sensitivity (80\%) but lower specificity (49\%) in diagnosing tendinopathy [4].

US examination is frequently used in emergency room settings as its accuracy for the detection of chronic ankle ligament injuries is very high (sensitivity of $98.9 \%$ and specificity of $96.2 \%$ ) [5]. The US assessment of hindfoot joints in patients with inflammatory arthritis of the ankle in the context of rheumatoid arthritis showed variable sensitivi- 
Figure 1: Normal radiographic appearance of ankle.
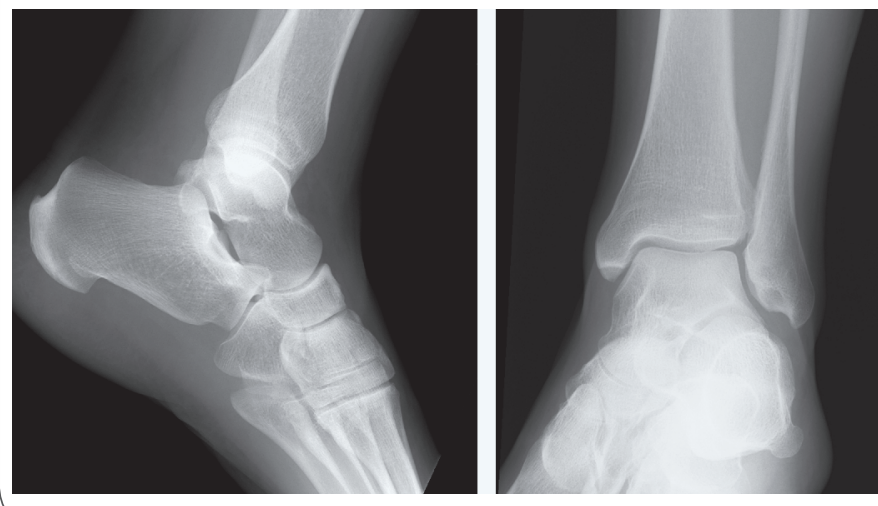

ty (64-89\%) and specificity (60-80\%) in a recent study [6].

The main abnormalities identifiable by US are: subcutaneous tissue oedema, ankle joint effusion associated with capsule extension, synovial hypertrophy (thickening), hyperperfusion due to abnormal vascularisation (positive Doppler signal), and structural abnormalities within the tendon, ligaments and bone surface (such as erosions, osteophytes) [7].

We opted for the US examination of our patient's ankles and this showed soft tissue oedema overlying the medial and lateral malleolus. PTT was thickened bilaterally and there was evidence of active tenosynovitis with Doppler signal on the right side (Figure 2). The posterior tibial nerve was also enlarged (Figure 3).

\section{Magnetic Resonance Imaging}

MRI is the most suitable technique to investigate subcutaneous inflammation, tendon and nerve abnormalities and can also offer an insight on early bone inflammatory changes such as bone marrow oedema, which is not amenable to US examination. $\mathrm{MRI}$ is considered the gold standard imaging technique for the diagnosis of inflammatory arthritis; therefore it is very difficult to assess its accuracy. Pre-arthroscopic assessment of patients with anterolateral ankle impingement reported huge variability of MRI sensitivity (39-100\%) and specificity (50-100) [8].
Figure 2: Longitudinal plan ultrasound examination of the posterior aspect of the medial malleolus. Evidence of tendinitis of the posterior tibial tendon with Doppler signal and fluid within the tendon sheath.

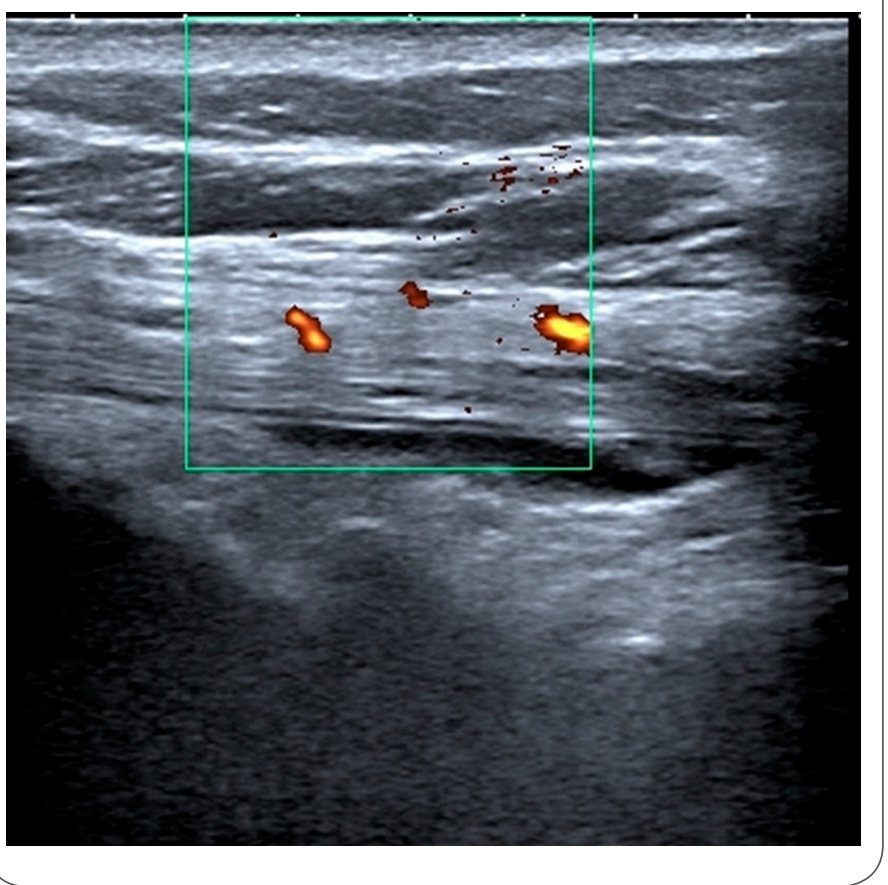

Figure 3: Enlarged tibialis posterior nerve on medial ankle transversal view ultrasound scan.

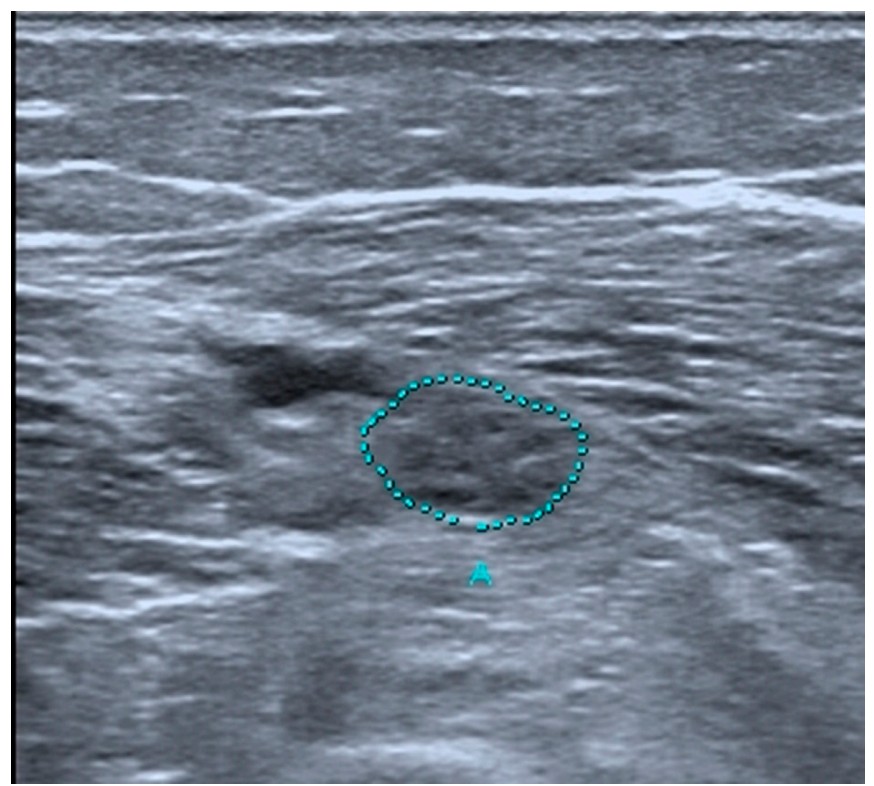


MRI and US are considered equally effective in detecting ankle soft tissue injuries [7]; however in a study in rheumatoid arthritis patients, MRI had a sensitivity of $50 \%$ compared to US for the detection of tenosynovitis, but similar sensitivity for the detection of synovitis; however, after the administration of contrast agent, MRI can reliably differentiate synovial inflammation from joint effusion [9]. Ligament abnormalities associated with ankle instability could also be identified by MRI, which also enables ankle laxity parameters measurements [10].

The most common MRI findings in patients with symptoms suggesting ankle impingement are: synovitis, fibrotic compression or scars, and bone prominences [11]. Acute neuropathic arthropathy of the ankle is usually associated with soft tissue oedema, bone marrow oedema and changes in the joint and bone morphology with associated collapse; however it is undistinguishable from osteomyelitis or septic arthritis [12]. MRI has an overall accuracy of $86 \%$ for the detection of peripheral nerve injuries with a sensitivity of $77 \%$ for posterior tibial nerve, $100 \%$ for medial plantar nerve, and $100 \%$ for lateral plantar nerve injury [13].

In our patient's case, the MRI scan confirmed the presence of enlarged tibialis posterior nerve with minimal ankle effusion and marked subcutaneous tissue inflammation (Figure 4).

\section{Other imaging studies}

Computer Tomography (CT) with tridimensional and multiplanar views may be used to evaluate joint and bone structural abnormalities. The sensitivity and specificity of CT vs. MRI in detecting bone marrow lesions was $90 \%$ and $80.5-81.6 \%$ respectively, according to a study from 2012 [14].

18F-fluorodeoxyglucose (FDG) positron emission tomography/computed tomography (PET) could also be used for detecting joint inflammation, infection and degenerative changes, owing to its very good sensitivity (up to 95\% for osteomyelitis) [15].
The authors therefore suggest combining one imaging method with great sensitivity with one with greater specificity for the assessment of joint inflammation, whenever an underlying systemic cause is suspicioned.

Figure 4: MRI scan showing markedly thickened tibialis posterior nerve just proximal and in the tarsal tunnel with soft tissue oedema (white arrow), small ankle effusion (grey arrow) and subcutaneos inflammation (white star).
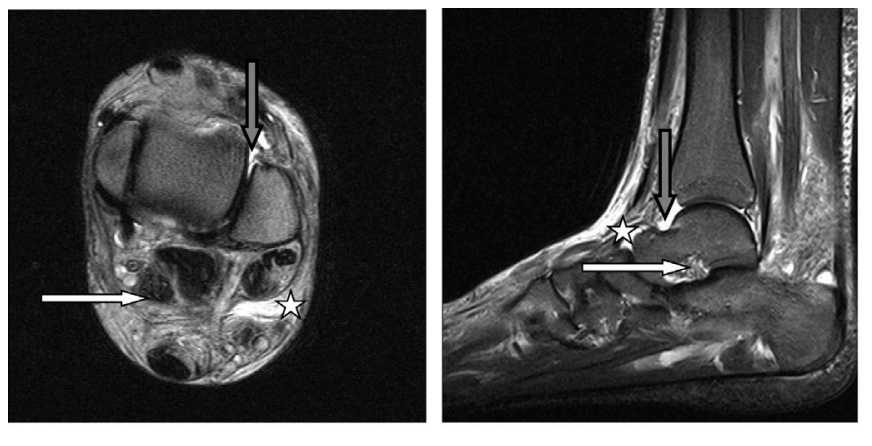

\section{Results}

Considering the patient's clinical picture and imaging evidence of peripheral nerve, tendon and subcutaneous inflammation, we proceeded with arranging a deep skin biopsy from the area of maximal inflammation, as assessed by the MRI scan. The patient was diagnosed with skin medium vessel necrotising vasculitis - polyarteritis nodosa - and started on Prednisolone $30 \mathrm{mg}$ daily. Her symptoms improved dramatically in a few weeks. To ensure the tapering of the steroid dose, Mycophenolate Mofetil 1 $\mathrm{g}$ twice daily was added. The ankle swelling almost disappeared after a couple of months of treatment and the skin discolouration diminished progressively. This is the first case report of peripheral nerve enlargement caused by small vessel vasculitis, which suggested that very rare diseases can have common clinical presentations. 


\section{References}

1. Nazarenko A, Beltran LS, Bencardino JT. Imaging evaluation of traumatic ligamentous injuries of the ankle and foot. Radiologic clinics of North America 2013; 51(3): 455-78.

2. McBride KL. Validation of the Ottawa ankle rules. Experience at a community hospital. Canadian family physician Medecin de famille canadien 1997; 43: 459-65.

3. Mei-Dan O, Kots E, Barchilon V, et al. A dynamic ultrasound examination for the diagnosis of ankle syndesmotic injury in professional athletes: a preliminary study. The American journal of sports medicine 2009; 37(5): 1009-16.

4. Khan KM, Forster BB, Robinson J, et al. Are ultrasound and magnetic resonance imaging of value in assessment of Achilles tendon disorders? A two year prospective study. British journal of sports medicine 2003; 37(2): 149-53.

5. Khoury V, Guillin R, Dhanju J, et al. Ultrasound of ankle and foot: overuse and sports injuries. Seminars in musculoskeletal radiology 2007; 11(2): 149-61.

6. Wakefield RJ, Freeston JE, O'Connor P, et al. The optimal assessment of the rheumatoid arthritis hindfoot: a comparative study of clinical examination, ultrasound and high field MRI. Annals of the rheumatic diseases 2008; 67(12): 1678-82.

7. Margetic P, Pavic R. Comparative assessment of the acute ankle injury by ultrasound and magnetic resonance. Collegium antropologicum 2012; 36(2): 605-10.

8. Haller J, Bernt R, Seeger T, et al. MR-imaging of anterior tibiotalar impingement syndrome: agreement, sensitivity and specificity of MR-imaging and indirect MR-arthrography. European journal of radiology 2006; 58(3): 450-60.

9. McQueen FM, Chan E. Insights into Rheumatoid Arthritis from Use of MRI. Curr Rheumatol Rep 2014; 16(1).

10. Seebauer CJ, Bail HJ, Rump JC, et al. Ankle laxity: stress investigation under MRI control. AJR American journal of roentgenology 2013; 201(3): 496-504.

11. Linklater J. MR imaging of ankle impingement lesions. Magnetic resonance imaging clinics of North America 2009; 17(4): 775800 , vii-viii.

12. Ergen FB, Sanverdi SE, Oznur A. Charcot foot in diabetes and an update on imaging. Diabetic foot \& ankle 2013; 4.

13. Burge AJ, Gold SL, Kuong $S$, et al. High-resolution magnetic resonance imaging of the lower extremity nerves. Neuroimaging clinics of North America 2014; 24(1): 151-70.

14. Guggenberger R, Gnannt R, Hodler J, et al. Diagnostic performance of dual-energy $\mathrm{CT}$ for the detection of traumatic bone marrow lesions in the ankle: comparison with MR imaging. Radiology 2012; 264(1): 164-73.

15. Gotthardt $M$, Bleeker-Rovers $C P$, Boerman $O C$, et al.I $m$ a g in $g$ of inflammation by PET, conventional scintigraphy, and other imaging techniques. Journal of nuclear medicine technology 2013; 41(3): 157-69.

\section{Comment on this article:}

\section{(f) in $8+\mathbf{S} P$}

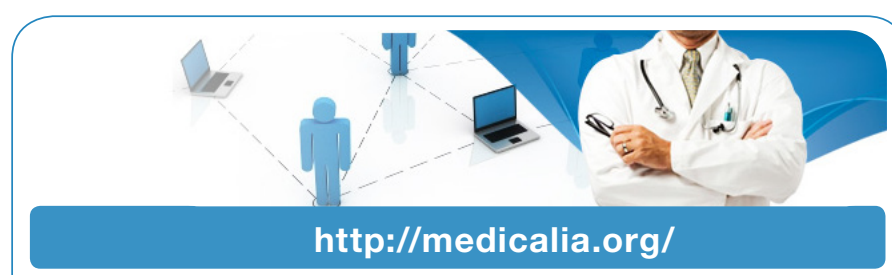

Where Doctors exchange clinical experiences, review their cases and share clinical knowledge. You can also access lots of medical publications for free. Join Now!

\section{Publish with iMedPub}

\section{http://www.imed.pub}

International Archives of Medicine is an open access journal publishing articles encompassing all aspects of medical science and clinical practice. IAM is considered a megajournal with independent sections on all areas of medicine. IAM is a really international journal with authors and board members from all around the world. The journal is widely indexed and classified Q1 in category Medicine. 